The final sample included 1125 newborns. The newborns were separated by groups based on the CDC recommendations. In 21 cases with a negative GBS screening $(n=814)$, the approach differed from the recommended and 10 cases needed admission in a Neonatal Intensive Care Unit (NICU).

In the group were GBS screening was not performed/ unknown, the recommended approach was performed in $41,9 \%$ and 3 cases needed admission in a NICU ( 1 for respiratory distress syndrome, 1 due to risk for infection and 1 for fetal growth restriction). When GBS screening was positive with a correct intrapartum prophylaxis, 96\% followed the recommendations. In the suspicion of chorioamnionitis all newborns were screened accordingly to the recommendations.

No cases of GBS early onset sepsis were found during the studied period.

Conclusion The rate of mothers tested for GBS colonization (96,9\%) was high, compared to similar studies, which could justify the absence of GBS related sepsis.

The group which led to a greater disagreement between the recommendation and the approach was the one with no/ unknow screening test. Results from this screening program should be analyzed frequently to verify its efficiency and the main difficulties in the approach.

\section{GP268 IMPACT OF THE USE OF ORAL DEXTROSE GEL FOR NEONATAL HYPOGLYCAEMIA AND ADMISSION RATES TO NEONATAL UNITS: A SYSTEMATIC REVIEW AND META-ANALYSIS}

${ }^{1}$ Grainne Egan*, ${ }^{1}$ Claire Thompson, ${ }^{2}$ Declan Patton, ${ }^{1,3}$ Roy K Philip. ${ }^{1}$ University Maternity Hospital Limerick (UMHL), Limerick, Ireland; ${ }^{2}$ Royal College of Surgeons in Ireland (RCSI), Dublin, Ireland; ${ }^{3}$ Graduate Entry Medical School (GEMS), University of Limerick, Limerick, Ireland

\subsection{6/archdischild-2019-epa.327}

Background Neonatal hypoglycaemia $(\mathrm{NH})$ is a leading cause of admission to neonatal units. It affects $15 \%$ of newborn infants and 50\% have risk factors. Dextrose gel (40\%), often described as oral gel, can be administered to buccal mucosa and lingual surfaces of infant's mouth resulting in a rapid absorption into the circulation. Rawat et al. (2016) recommended that treatment with dextrose gel is noninvasive, inexpensive and easy to administer.

Objective To ascertain the impact of $40 \%$ dextrose oral gel on documented neonatal hypoglycaemia in the postnatal wards to prevent neonatal admission rates.

Method A systematic review (SR) of quantitative studies in medical databases including; Medline, Cumulative Index to Nursing and Allied Health Literature (CINAHL), Embase and SCOPUS were searched in February 2018. Grey literature was searched for full-text conference papers in Irish Health Repository (LENUS) and the National Institute for Health and Care Excellence (NICE) inclusions. Using combinations of the relevant key words incorporating the PICO model ('dextrose gel', 'glucose gel', 'neonate', 'newborn', 'hypoglycaemia' and 'admission'), review was performed and English language limitation was applied. Quantitative studies that did not measure neonatal admission rates were excluded. Search results were reported following the PRISMA guidelines. Data from eligible studies were synthesized for a meta-analysis. A dichotomous analysis was preformed through Review Manger 5 software (RevMan 5.3) to give a forest plot and funnel plot on the primary outcome as admission rates to a neonatal unit.

Results The search yielded 130 papers, including 128 from database search and two from grey literature. 21 duplicates were removed leaving 109 papers for screening and of these 19 full text articles were eligible for initial review. Five studies were identified fulfilling the predetermined criteria and included for analysis. Two randomised control trials (RCT's) were analysed and the meta-analysis shows that the use of $40 \%$ dextrose gel for neonatal hypoglycaemia decreases admission rates for at risk infants. Three quantitative studies of other designs also reported a decrease in admission rates for hypoglycaemia. Increased rate of exclusive breastfeeding was also consistently linked to the use of oral application of dextrose gel across the five studies.

Conclusion Dextrose gel (40\%) as an oral mucosal application can be safely and effectively used as a method of treating neonatal hypoglycaemia in at risk infants in the postnatal wards, thus reducing admission to the neonatal unit. Secondary benefits of reduced mother-infant separation and improved breastfeeding also were noted.

\section{GP269 ARE READMISSIONS FROM HOME POTENTIALLY PREVENTABLE?}

Anandi Nayana Singh, Shree Vishna Rasiah*. Birmingham Women's and Children's NHS Foundation Trust, Birmingham, UK

\subsection{6/archdischild-2019-epa.328}

Background There is a national strategy ATAIN aimed at reducing term admissions to the neonatal unit. Whilst this is aimed at looking at potentially preventable term admissions to the neonatal unit, it does not address the issue of re-admission following discharge from the postnatal ward or neonatal unit. Given the limited midwifery resources in the community, we believe some of the re-admissions are potentially preventable if there were adequate resources to support the mother and baby at home.

Aims To review the reasons for readmissions from home and identify potentially avoidable causes.

Methods We retrospectively reviewed the BadgerNet neonatal electronic patient record to identify readmissions over the last financial year between 01/04/2017 and 31/03/2018. A standard excel profoma was used to collect and analyse the data. The reason for admission, interventions carried out and outcome was reviewed including the length of stay.

Results In the last financial year there were 142 babies resulting in 143 readmissions to the postnatal or transitional care ward. Of these 120 babies were re-admitted for jaundice as a primary cause of admission. Of these jaundiced babies, 31 also had associated weight loss noted on admission. One baby had two episodes of re-admission for jaundice. Five of these babies had an infection screen, which were negative and antibiotics were discontinued by $48 \mathrm{hrs}$. The median length of stay was 4 days and the range was between 3 and 11 days. Only one had admission to the neonatal unit with bronchiolitis. The total length of stay for the 120 babies was 446 days.

Another 22 babies were readmitted because of weight loss. The median length of stay was 3 days and the range was between 1-4 days. The total length of stay for the 22 babies 
was 70 days. Of all the re-admission, 99 were breastfeeding on re-admission, 24 were mixed feeds and 19 bottle fed.

Conclusions The two main reasons for readmissions were jaundice and weight loss. There was an overlap of this because a proportion of jaundiced babies were noted to have weight loss or poor feeding on readmission. We believe that these are potentially avoidable readmissions if there was sufficient feeding support for the mother and babies in the community. It would alleviate the additional emotional stress imposed on the mother and family by the readmission. It would also reduce the impact on the bed occupancy on an already stretched maternity and transitional care wards.

\section{GP270 HEALTH PROBLEMS IN NEONATES WITH PERINATAL HIV EXPOSURE}

${ }^{1}$ Svetlana Shugaeva, ${ }^{2}$ Alla Petrova, ${ }^{2}$ Anastasiya Vanyarkina*, 2 Lyubov Rychkova. ${ }^{2}$ rrkutsk State Medical University, Irkutsk, Russian Federation; ${ }^{2}$ Scientific Centre for Family Health and Human Reproduction Problems, Irkutsk, Russian Federation

10.1136/archdischild-2019-epa.329

Introduction Antenatal and perinatal periods determine the potential of a child's health for near and distant future. Considering the increasing number of HIV-exposed uninfected newborns (especially in areas of high risk of HIV infection), the aim of our study was to determine the prevalence of health problems of newborns who avoided perinatal HIV transmission.

Methods We conducted a cohort randomized study that included 177 children from birth to 18 months of life. The sample was divided in two groups, equivalent in sex: the main group ( $\mathrm{n}=87$, random sample) comprised neonates with perinatal HIV exposure, the comparison group included children with no HIV exposure (matched sample). Exclusion criteria were confirmed HIV infection $(\mathrm{n}=3)$, and parents' refusal to participate $(\mathrm{n}=0)$. We evaluated the frequency $\left(\mathrm{P}_{\mathrm{abs}} / \mathrm{P} \%\right)$ of seven signs (prematurity, intrauterine growth retardation, neonatal abstinence syndrome, abnormalities of postnatal adaptation, perinatal damage of CNS, congenital and perinatally-acquired infections, congenital malformations and/or minor developmental abnormality). A comparative analysis was performed using the $\chi 2$ criterion and its modifications (Yates corrected Chi-square, two-tailed Fisher exact p), relative risk index was calculated (RR [CI0.95]).

Results Health problems in neonates according to the analyzed signs were significantly more frequent in main group (76/ $87.6 \%)$ than in comparison group $(28 / 31.1 \%) \quad(\mathrm{RR}=2.8$ $[2.0-3.9] ; \chi 2=57.75, \mathrm{p}<0.0001)$.

In contrast with children, not exposed to HIV, children of HIV-infected mothers showed statistically significant incidence of the following signs: intrauterine growth retardation: 26/ $29.9 \%$ vs $5 / 5.5 \% \quad(\mathrm{RR}=5.4[2.2-13.4] ; \chi 2=16.5, \mathrm{p}$ $<0.0001)$; abnormalities of postnatal adaptation: $14 / 16.1 \%$ vs $3 / 3.3 \%(\mathrm{RR}=4.8[1.4-16.2] ;-, \mathrm{p}=0.005)$; congenital and perinatally-acquired infections: $17 / 19.5 \%$ vs $7 / 7.8 \% \quad(\mathrm{RR}=$ $2.5[1.1-5.7] ; \chi 2=5.2, \mathrm{p}=0.022)$; neonatal abstinence syndrome $12 / 13.8 \%$ vs $0 / 0 \% \quad(\mathrm{RR}=25.9$ [1.6-430.1] - calculated by L.A. Pevnitsky's method; -, p <0.0001); perinatal CNS damage: $30 / 34.5 \%$ vs $15 / 16.7 \% \quad(\mathrm{RR}=2.1 \quad[1.2-3.6]$; $\left.\chi^{2}=7.4, \mathrm{p}=0.007\right)$.

The most severe problems (frequency of occurrence and the number of registered conditions) were found in HIV- exposed children in families with low income and families where adults had alcohol and/or drug dependence.

Conclusions Health problems, relating to disorders in intrauterine growth and early postnatal period are registered mostly in HIV-exposed uninfected children, born to HIV-positive mothers. Increased incidence of health problems and their concentration in low-income families with deviant lifestyle requires careful approach by pediatricians.

\section{GP271 AN AUDIT OF A TERTIARY NEONATAL CENTRE'S TRANSITION FROM INDIVIDUALISED TO STOCK PARENTERAL NUTRITION IN EXTREMELY PRETERM INFANTS}

James Trayer* , Anne Twomey. National Maternity Hospital, Dublin, Ireland

10.1136/archdischild-2019-epa.330

Introduction Infants $\leq 28$ weeks gestational typically require parenteral nutrition (PN) while enteral feeds are slowly advanced. The National Maternity Hospital has recently commenced prescribing standardised $\mathrm{PN}$ for these infants (using new and more concentrated stock bags that are produced by Baxter Healthcare Ltd) rather than individualised PN (IPN). Our aim was to assess the clinical impact of this change.

Methods A retrospective chart review was conducted including all infants $\leq 28$ weeks gestation during a six month period from August 2018 to January 2019. The outcomes reviewed included subsequent need for IPN, electrolyte abnormalities, need for additional intravenous fluids to correct biochemical abnormalities, proportion of infants receiving the recommended nutritional requirements.

Results A total of 21 babies were included in the study. 7/21 (33\%) required a switch from SPN to IPN. The most common reason was hyponatraemia (3/7) followed by hypoglycaemia (2/7). Electrolyte abnormalities were seen in $13 / 21$ (62\%). The most common reason was hyponatraemia $(8 / 21)$ followed by hypernatraemia (4/21). Electrolyte abnormalities were also more commonly seen in infants <25wks $(7 / 8$, $88 \%)$. Supplemental fluids were required in 6/21 (29\%) babies - 3 for hyponatraemia and 2 for hypernatraemia. Daily targeted volumes of SPN to optimise nutrition were frequently missed, especially on D1, when only $2 / 21(10 \%)$ infants received the recommended volumes. This was largely due to a hospital based practice of using non-nutritive fluids to maintain the patency of the second lumen of an umbilical venous line $(12 / 21,57 \%)$. Other reasons included the need for fluid restriction $(5 / 21,24 \%)$ or the need for inotropes $(2 / 21,10 \%)$.

Conclusions and discussion The switch to SPN has reduced the need for IPN from $100 \%$ to $33 \%$. This represents a significant reduction in time spent prescribing IPN, a complex product associated with a high risk of prescription errors. The reduced reliance on IPN also represents a financial saving for the hospital. This audit highlighted the significant impact that a hospital based practice used to maintain patency of umbilical lines has on our ability to give optimal nutrition (both SPN and IPN) during the first few days of life. This practice now needs to be re-assessed in the light of this finding. Due attention also needs to be given sodium requirements when using these new SPN bags to avoid the risk of hypo/hypernatraemia. 